\section{Revisão sistemática da eficácia do interferon alfa (convencional, peguilado) e lamivudina para o tratamento da hepatite crônica B}

\author{
Efficacy of interferon (conventional, pegylated) and \\ lamivudine for treatment of chronic hepatitis B: \\ a systematic review
}

\author{
1 Faculdade de Medicina, \\ Universidade Federal de \\ Minas Gerais, Belo Horizonte, \\ Brasil. \\ 2 Faculdade de Farmácia, \\ Universidade Federal de \\ Minas Gerais, Belo Horizonte, \\ Brasil. \\ Correspondência \\ A. M. Almeida \\ Departamento de Medicina \\ Preventiva e Social, \\ Faculdade de Medicina, \\ Universidade Federal de \\ Minas Gerais. \\ Rua Prof. Antônio Aleixo 760, \\ apto. 602, Belo Horizonte, $M G$ \\ 30180-150, Brasil. \\ alessandraalm@gmail.com
}

\begin{abstract}
Chronic hepatitis $B$ is considered a major public health problem, and its treatment entails increasing health budget expenses with high-cost drugs covered by Unified National Health System. The objective of this study was to compare the efficacy of interferon (conventional; pegylated - PEG2a) and lamivudine (LAM) for the treatment of chronic hepatitis $B$ through a systematic review, selecting randomized, controlled clinical trials identified in PubMed and LILACS. Target outcomes were virological, biochemical, and histological response, seroconversion, and adverse effects. The review selected 35 articles. Presence or absence of HBeAg and pre-treatment alanine aminotransferase (ALT) levels were considered important factors in the initial therapeutic indication. Treatment with conventional interferon enables lasting disease inactivation and can result in HBsAg seroconversion. PEG2a showed better efficacy than interferon and LAM and similar side effects to interferon. LAM presents advantages such as its sensitivity in the HbeAg-negative phenotype, while its main disadvantage is the development of resistance.
\end{abstract}

Chronic Hepatitis B; Interferons; Lamivudine; Treatment Outcomes

\author{
Alessandra Maciel Almeida ${ }^{1}$ \\ Dirce Inês da Silva 2 \\ Augusto Afonso Guerra Jr. 1 \\ Grazielle Dias Silva 1 \\ Francisco de Assis Acurcio 1,2
}

\section{Introdução}

A hepatite crônica B constitui um grave problema de saúde pública, pois afeta mais de 400 milhões de pessoas no mundo, sendo contabilizado mais de um milhão de mortes anualmente 1 . Esses pacientes apresentam risco aumentado de desenvolvimento de cirrose, hepatite descompensada e carcinoma hepatocelular 2 O objetivo do tratamento é alcançar a supressão contínua da replicação do vírus da hepatite B (HBV) e remissão da doença do fígado ${ }^{3}$.

O tratamento para hepatite B depende de vários fatores, como o estágio da doença, a presença ou ausência do antígeno "e", o potencial de resistência ao medicamento e a subseqüente incapacidade de utilização de um medicamento, em particular nos estágios finais da doença crônica do fígado. Esses e outros fatores são importantes para avaliar o momento de início de tratamento, o tipo de tratamento indicado e sua duração 4 .

Entre as alternativas medicamentosas preconizadas nos protocolos clínicos e diretrizes terapêuticas para medicamentos excepcionais do Ministério da Saúde 5, o interferon alfa 2a e interferon alfa $2 \mathrm{~b}$ (IFN 2a e 2b) tem sido utilizados, por muitos anos, como primeira escolha de tratamento com baixos níveis de HBV do DNA, altos níveis de alanina amino transferase (ALT) e com biópsia hepática ativa. A meta do tratamento é ativar resposta imunológica em termos de soroconversão HBeAg, queda ou negativação 
dos níveis de DNA do HBV e, quando possível, soroconversão HBsAg. Essa forma de tratamento é a primeira opção para alterar o sistema imunológico, visando a obter a eliminação ou remissão da doença. Para o tratamento de longo-prazo ou manutenção, nos casos de falha do tratamento com o interferon alfa ou quando a doença avançou, geralmente utiliza-se lamivudina (LAM), que é um análogo nucleosídeo/nucleotídeo. Essa forma de tratamento é a opção de escolha para os pacientes HBeAg negativo, com elevados níveis de DNA do HBV e ALT. Nesses pacientes, a utilização de análogos nucleosídeo/nucleotídeo é necessária para a obtenção da supressão em longo prazo da replicação de HBV 4.

O mais novo "peguilado" derivado de alfa interferon tornou-se disponível recentemente. O interferon peguilado 2a (PEG2a) apresenta como vantagens o prolongamento do efeito biológico e a necessidade de menos aplicações, comparado ao convencional. Contudo, o PEG2a não deve ser utilizado como terapia de manutenção devido aos efeitos adversos (sendo menos tolerado em longo prazo) e é contra-indicado em pacientes com doença hepática descompensada.

O objetivo deste estudo, utilizando método de revisão sistemática de estudos experimentais randomizados e controlados, foi comparar a eficácia dos medicamentos preconizados no Protocolo Clínico e Diretrizes Terapêuticas da Hepatite Viral Crônica B do Ministério da Saúde 5 para o tratamento da hepatite viral crônica B: interferon e LAM.

\section{Material e método}

Foi conduzida uma pesquisa sobre artigos publicados nas bases de dados MEDLINE (PubMed) e Literatura Latino-Americana e do Caribe em Ciências da Saúde (LILACS) para identificar estudos relevantes, no período de janeiro de 1970 a dezembro de 2007. Para a busca no PubMed, utilizou-se o programa Reference Manager 11 (Thomson ISI ResearchSoft, Estados Unidos). A busca no LILACS foi realizada no portal BIREME (BVS) e os resultados foram importados para esse mesmo programa. Também foram incluídas as bases de dados Cochrane Controlled Trials Databases e NHS Centre for Reviews and Dissemination.

Selecionaram-se estudos que abordaram a história natural da infecção crônica pelo HBV, definição da resposta virológica, relação entre resposta virológica, resistência e estado de saúde subseqüente, efeitos adversos, bem como a eficácia dos medicamentos utilizados para o tratamento da hepatite crônica B.
A revisão sistemática considerou estimativas sobre: (a) condições clínicas relevantes da infecção crônica pelo HBV; (b) a eficácia das estratégias de tratamento preconizadas nos protocolos clínicos e diretrizes terapêuticas para medicamentos excepcionais do Ministério da Saúde para a hepatite crônica $\mathrm{B}$.

O processo de busca utilizou as seguintes palavras-chave: "Liver" OR "figado", "chronic hepatitis B virus" OR "chronic HBV infection” OR "hepatite crônica B", "Interferon alfa" OR "interferon-alfa" OR "lamivudine" OR "lamivudina" OR "pegylated interferon alfa-2a" OR "PEG” OR "Cirrhosis" OR "cirrose" OR "hepatocellular carcinoma” OR "carcinoma hepatocelular" OR "liver transplant” OR "transplante de fígado" AND "humans" AND "english" OR "inglês" OR "spanish" OR "espanhol” OR "portuguese” OR "português" NOT "Children” OR "crianças".

Foram adotados os seguintes critérios de inclusão dos estudos: (a) intervenções - medicamentos utilizados isoladamente ou em combinação (IFN 2a e 2b, LAM, PEG2a); (b) pacientes - adultos com infecção crônica pelo HBV, incluindo pacientes HBeAg positivo e negativo com doença compensada e descompensada do fígado; (c) tipos de estudos - clínicos randomizados e controlados.

Os critérios adotados para a exclusão dos estudos foram: (a) aqueles que não estavam no idioma inglês, português ou espanhol; (b) que não eram realizados em humanos; (c) limitados a crianças e adolescentes; (d) que não eram relacionados à hepatite crônica $\mathrm{B}$; (e) relacionados somente a doenças colestáticas do fígado; (f) relacionados a doenças auto-imunes do fígado; (g) relacionados a doenças metabólicas do fígado.

Inicialmente, para verificar se os artigos atendiam aos critérios de inclusão, os títulos de todos os estudos identificados foram avaliados. $\mathrm{Na}$ segunda fase, os resumos foram analisados por dois revisores independentes. Os estudos em que não houve concordância quanto à inclusão foram analisados por um terceiro revisor.

Foram selecionados os ensaios clínicos randomizados e controlados que incluíam comparação de medicamentos utilizados para o tratamento da hepatite crônica B com placebo, outro medicamento ou o melhor cuidado de suporte.

As medidas de resultado consideradas foram: resistência ao medicamento, resposta histológica (resultados de biópsia como necroinflamação/fibrose), resposta bioquímica (níveis de ALT), resposta virológica (taxa de soroconversão e replicação viral - DNA do HBV), soroconversão (perda de $\mathrm{HBeAg}$ /anti HBe, perda de HBsAg/anti HBs) e efeitos adversos. 
Para avaliação da qualidade metodológica dos estudos, foi utilizada a escala de JADAD modificada ${ }^{6}$. O sistema de JADAD consta de três tópicos centrados na validade interna da pesquisa, contendo sete questões dicotômicas, referentes à randomização, mascaramento e perda de participantes, diretamente relacionadas com a redução de vieses. Estudos clínicos com pontuação 0 a 2 são tidos como de baixa qualidade, de 3 a 4 de moderada qualidade e 5 a 6 de alta qualidade.

Para o processo de avaliação da qualidade das publicações, também foi proposta a participação de dois revisores independentes e de um terceiro revisor quando não houvesse consenso.

Os artigos selecionados foram organizados em tabela, de acordo com autor e ano de publicação, estado de HBeAg, número de participantes (N), tempo do estudo (Te) e tempo de seguimento (Ts), protocolo de tratamento e escores da escala de JADAD modificada.

\section{Resultados}

A estratégia de pesquisa identificou 525 títulos e resumos. Foram incluídos 317 títulos e após análise do terceiro revisor, 14 títulos foram excluídos, permaneceram 303 resumos para análise. Desses, 262 foram excluídos pelas seguintes razões: revisão de literatura, relação com a história natural da doença, resumos de conferências, estudos observacionais (caso-controle, coorte, transversais, estudos de caso), estudos de custos, outros medicamentos para o tratamento da hepatite crônica B, outras hepatites e textos duplicados. Foram selecionados 41 artigos sobre ensaios clínicos randomizados e controlados. Ainda nessa fase, foram excluídos seis ensaios clínicos que se referiam apenas à comparação de doses distintas dos medicamentos.

Dos resumos incluídos, foram selecionados 35 artigos: 18 abordando eficácia do interferon alfa (Tabela 1), três do interferon peguilado (Tabela 2) e 14 da LAM (Tabela 3). A maioria dos estudos apresentou qualidade moderada na classificação da escala de JADAD modificada. Estudos enfocando o interferon foram classificados como de moderada a baixa qualidade. Investigações com o interferon peguilado apresentaram qualidade moderada e aqueles com a LAM foram avaliados como de moderada a alta qualidade. A concordância entre os revisores, obtida pelo método kappa ponderado, foi considerada alta $(0,82)$.

\section{IFN 2a e $2 b$}

Foram analisados os estudos clínicos randomizados e controlados utilizando IFN 2a e $2 \mathrm{~b}$ para o tratamento da hepatite crônica $\mathrm{B}$ em indivíduos HBeAg positivo e doença do fígado compensada e também em pacientes HBeAg negativo com níveis elevados de DNA do HBV, HBsAg positivo e presença de anti-HBe. Os resultados primários considerados foram a perda de HBeAg, DNA do HBV e HBsAg. Os resultados secundários foram o desenvolvimento de anticorpos anti-HBs e antiHBe, normalização dos níveis ALT e melhoria dos índices histológicos. Além disso, consideraramse como critério de exclusão os pacientes HIV positivo e com hepatite C. Os resultados foram estratificados para HBeAg positivo e negativo devido ao curso da doença, prognóstico e diferentes respostas às alternativas medicamentosas, considerando-se os resultados com diferenças estatisticamente significantes $(\mathrm{p}<0,05)$.

Observou-se que com a utilização do IFN 2a e $2 \mathrm{~b}$ em pacientes HBeAg positivo com tempo de seguimento de pelo menos seis meses, a perda de atividade da replicação viral foi melhor no grupo dos tratados comparada à perda espontânea dos marcadores virais em controles. Foi demonstrado efeito de redução de DNA do HBV durante o tratamento de 4 a 6 meses (16\%-50\% vs. 5\%$19 \%) 7,8,9,10,11$ e no seguimento de até quatro anos (19\%-61\% vs. 22\%) 8,9. A perda de HBeAg encontrada foi de $11 \%-50 \%$ vs. 5\%-11\% 7,8,9,10,11,12,13 em seis meses de tratamento. Respostas ao tratamento com interferon foram observadas em 33\% após seis meses de tratamento 10,11,12,13,14 e com perda de HBsAg destacaram-se em seis meses de tratamento taxas de $16 \%$ nos tratados e soroconversão natural de $0 \% 8$.

Quando os resultados secundários foram analisados, o tratamento com interferon apresentou desenvolvimento de anticorpos antiHBe em 35\% 11 e normalização dos níveis de ALT em $5 \%-40 \%$ vs. $0 \%-13 \%$, sendo que os pacientes com níveis elevados de ALT pré-tratamento (> 2 vezes o nível normal) apresentaram maiores reduções dos níveis de ALT 8,11. Outros autores não demonstraram normalização dos níveis de ALT 15,16. Os índices histológicos exibiram decréscimo dos escores, representando reduções em torno de $50 \%$ 11,13,15. Na maioria dos estudos não foi observado o desenvolvimento de cirrose durante o tratamento.

A dosagem e o tempo ótimo de duração do tratamento do interferon são controversos. A administração da droga por duas vezes/semana apresentou resultados inferiores aos encontrados em administração de três vezes 7. Doses mais elevadas mostraram-se mais efetivas que as mais baixas 13,15 ou não houve diferenças entre os grupos tratados em diferentes dosagens 16 . Contudo, doses altas estão associadas ao aumento da freqüência e à gravidade dos efeitos 
Informações sobre os artigos selecionados e o escore da escala de JADAD modificadas para estudos clínicos randomizados e controlados de interferon alfa 2a e interferon alfa $2 b$ (IFN 2a e 2b).

\begin{tabular}{|c|c|c|c|c|c|c|c|}
\hline Estudo & Ano & $\begin{array}{l}\text { Estado de } \\
\text { HBeAg }\end{array}$ & $\mathbf{N}$ & $\begin{array}{c}\text { Tempo de } \\
\text { estudo }\end{array}$ & $\begin{array}{l}\text { Tempo de } \\
\text { seguimento }\end{array}$ & $\begin{array}{l}\text { Protocolo de } \\
\text { tratamento }\end{array}$ & $\begin{array}{l}\text { Escore da escala } \\
\text { de JADAD } \\
\text { modificada }\end{array}$ \\
\hline Hadziyannis et al. 19 & 1990 & Negativo & 469 & 12 meses & $\begin{array}{l}\text { Média: } \\
5 \text { anos }\end{array}$ & $\begin{array}{l}\text { IFN 2a 2,5UM; 5,0UM, } \\
\text { 10UM; IFN 2a 1,5UM e } \\
\text { 18UM; IFN 2a 4,5UM }\end{array}$ & 2 \\
\hline Fattovich et al. 17 & 1992 & Negativo & 60 & 12 meses & 18 meses & IFN 5UM & 3 \\
\hline Lampertico et al. 18 & 1997 & Negativo & 42 & 24 meses & 3 anos & IFN 2b; 6,0UM & 2 \\
\hline Carreño et al. 7 & 1987 & Positivo & 20 & 6 meses & & rIFN 2b; 5,5MU & 3 \\
\hline Krogsgaard 44 & 1998 & Positivo & 469 & 12 meses & $\begin{array}{l}\text { Média: } \\
5 \text { anos }\end{array}$ & $\begin{array}{c}\text { IFN 2a; 2,5UM, 5,0UM, } \\
\text { 10UM; IFN alfa-2a 1,5MU e } \\
\text { 18UM; IFN 2a 4,5UM }\end{array}$ & 3 \\
\hline Porres et al. 13 & 1988 & Positivo & 24 & 6 meses & 15 meses & $\begin{array}{l}\text { rIFN 2a; } 2,5 U M / m^{2} ; \\
\text { rIFN 2a; } 25,0 U M / m^{2} ; \\
\text { rIFN 2a; } 10 U M / m^{2}\end{array}$ & 2 \\
\hline Lok et al. 9 & 1988 & Positivo & 72 & 12 meses & 24 meses & $\begin{array}{l}\text { rIFN 2a 2,5UM; } \\
\text { rIFN 2a 5,0UM; } \\
\text { rIFN 2a 10UM }\end{array}$ & 3 \\
\hline Müller et al. 10 & 1990 & Positivo & 58 & 12 meses & 6 meses & rIFN 2b; 3,0UM & 2 \\
\hline Pastore et al. 12 & 1992 & Positivo & 18 & 6 meses & 24 meses & IFN 2b; 5,0UM & 2 \\
\hline Di Bisceglie et al. 8 & 1993 & Positivo & 47 & 6 meses & 2 a 4 anos & IFN 2b; 10UM & 3 \\
\hline Carreño et al. 14 & 1999 & Positivo & 57 & 4 anos & - & IFN 2; 4,5-18UM & 2 \\
\hline Fattovich et al. 45 & 1989 & Positivo & 33 & 16 meses & - & $\begin{array}{c}\text { IFN 2a; } \\
4,5 \text { mega unidades }\end{array}$ & 3 \\
\hline Perillo et al. 15 & 1990 & Positivo & 43 & 16 semanas & 5 anos & $\begin{array}{c}\text { IFN 2a: dose de } 5 \text { milhões e } \\
\text { prednisona } 20 \mathrm{mg}, 40 \mathrm{mg} \text { e } 60 \mathrm{mg}\end{array}$ & 3 \\
\hline Realdi et al. 46 & 1990 & Positivo & 82 & 16 semanas & - & IFN 2a; 4,5UM & - \\
\hline Thomas et al. 16 & 1994 & Positivo & 238 & 12 meses & - & $\begin{array}{l}\text { IFN 2a 2,5 mil; } \\
\text { IFN 2a 5,0 mil; } \\
\text { IFN 2a } 10 \mathrm{mil}\end{array}$ & 3 \\
\hline Harry et al. 47 & 1999 & Positivo & 469 & $\begin{array}{l}12 \text { a } 24 \text { semanas } \\
\text { (grupo I), } 16 \text { semanas } \\
\text { (grupos II e III) }\end{array}$ & 12 meses & 2,5UM; 5,0UM; 10UM & 4 \\
\hline Sarin et al. 11 & 1996 & Positivo & 30 & - & - & IFN 2b 2,5UM & 1 \\
\hline Lau et al. 48 & 1997 & Positivo & 45 & 16 semanas & 2 anos & IFN 2b 5UM; IFN 2b 10UM & 1 \\
\hline
\end{tabular}

UM: unidades por milhão; rIFN: interferon recombinante.

adversos 13,15. Os efeitos adversos mais freqüentes para o interferon convencional em HBeAg positivo foram febre, síndrome $f l u$, mialgia, artralgia, fadiga 9,12,13. Também foram detectados sintomas como leucopenia e trombocitopenia 8 .

Para pacientes HBeAg negativo utilizando o IFN 2a e 2b, foi observada redução na replicação viral, com reduções de DNA do HBV no tratamento de seis meses ( $63 \%$ vs. $20 \%)$ e no seguimento de 18 meses (53\% vs. 27\%) 17. Quando o tratamento foi suspenso, observou-se recidiva em $27 \%$ vs. 13\%, contudo, as diferenças não fo- ram estatisticamente significantes 17 . Resposta completa ao tratamento, relacionada à negativação de DNA do HBV e normalização dos níveis de ALT ocorreu em $60 \%$ dos pacientes comparada a nenhum paciente no grupo controle e se sustentou após 12 meses de tratamento. Quando os resultados secundários foram analisados, verificou-se redução nos níveis de ALT de 12 a 24 meses de tratamento (38\%-57 vs. $10 \%)$ e no seguimento de até três anos (28\%-53\% vs. 0\%) 17,18 . Melhorias histológicas foram constatadas em aproximadamente $50 \%$ dos pacientes no trata- 
Informaç̃̃es sobre os artigos selecionados e o escore da escala de JADAD modificadas para estudos clínicos randomizados e controlados de interferon peguilado 2a (PEG2a).

\begin{tabular}{|c|c|c|c|c|c|c|c|}
\hline Estudo & Ano & $\begin{array}{c}\text { Estado de } \\
\text { HBeAg }\end{array}$ & $\mathbf{N}$ & $\begin{array}{l}\text { Tempo de } \\
\text { estudo }\end{array}$ & $\begin{array}{l}\text { Tempo de } \\
\text { seguimento }\end{array}$ & $\begin{array}{l}\text { Protocolo de } \\
\text { tratamento }\end{array}$ & $\begin{array}{c}\text { Escore da escala } \\
\text { de JADAD } \\
\text { modificada }\end{array}$ \\
\hline Cooksley et al. 21 & 2003 & Positivo & 194 & 24 semanas & 24 semanas & 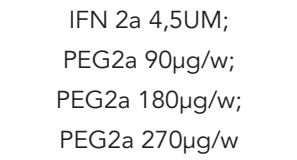 & 3 \\
\hline Marcellin et al. 22 & 2004 & Negativo & 552 & 48 semanas & 24 semanas & $\begin{array}{c}\text { PEG2a } 180 \mu \mathrm{g} / \mathrm{w} ; \\
\text { PEG2a } 180 \mu \mathrm{g} / \mathrm{w}+\text { LAM; } \\
\text { LAM }(100 \mathrm{mg} / \mathrm{dia})\end{array}$ & 4 \\
\hline Lau et al. 20 & 2005 & Positivo & 814 & 48 semanas & 24 semanas & $\begin{array}{c}\text { PEG2a } 180 \mu \mathrm{g} / \mathrm{w} ; \\
\text { PEG2a } 180 \mu \mathrm{g} / \mathrm{w}+\text { LAM; } \\
\text { LAM }(100 \mathrm{mg} / \mathrm{dia})\end{array}$ & 4 \\
\hline
\end{tabular}

IFN 2a: interfe-ron alfa 2a; LAM: lamivudina.

Tabela 3

Informações sobre os artigos selecionados e o escore da escala de JADAD modificadas para estudos clínicos randomizados e controlados de lamivudina (LAM).

\begin{tabular}{|c|c|c|c|c|c|c|c|}
\hline Estudo & Ano & $\begin{array}{l}\text { Estado de } \\
\text { HBeAg }\end{array}$ & $\mathbf{N}$ & $\begin{array}{l}\text { Tempo de } \\
\text { estudo }\end{array}$ & $\begin{array}{c}\text { Tempo de } \\
\text { seguimento }\end{array}$ & $\begin{array}{l}\text { Protocolo de } \\
\text { tratamento }\end{array}$ & $\begin{array}{c}\text { Escore da escala } \\
\text { de JADAD } \\
\text { modificada }\end{array}$ \\
\hline Perrillo et al. 23 & 2002 & Positivo & 805 & 16 semanas & 52 semanas & $\begin{array}{l}\text { LAM (100mg); } \\
\text { IFN 2b 10UM; } \\
\text { LAM (100mg) + } \\
\text { IFN 2b 10UM }\end{array}$ & 3 \\
\hline Manolokopoulos et al. 32 & 2003 & Negativo & 60 & 2 anos & 3 anos & LAM (100mg) & 2 \\
\hline Naoumov et al. 36 & 2001 & Negativo & 24 & 52 semanas & 52 semanas & LAM (100mg) & 2 \\
\hline Dienstag et al. 26 & 2003 & Positivo & 40 & 3 anos & 5 anos & LAM (100mg) & 4 \\
\hline Lau et al. 24 & 2000 & Positivo/Negativo & 27 & 24 a 47 meses & 5 anos & LAM (100mg) & 3 \\
\hline Leung et al. 31 & 2001 & Positivo & 58 & 2 anos & 3 anos & LAM (100mg) & 5 \\
\hline Fontana et al. 38 & 2001 & Negativo & 33 & 61 semanas & 85 semanas & LAM (100mg) & 2 \\
\hline Lok et al. 30 & 2003 & Positivo & 998 & 4 anos & 6 anos & LAM (100mg) & 4 \\
\hline Andreone et al. 37 & 2004 & Negativo & 22 & 42 meses & 3 anos & LAM (100mg) & 2 \\
\hline Buti et al. 25 & 2001 & Positivo & 60 & 2 anos & - & LAM (100mg) & 3 \\
\hline Fung et al 33 & 2004 & Positivo & 50 & 2 anos & 3 anos & LAM (100mg) & 2 \\
\hline Barbaro et al. 29 & 2001 & Positivo & 151 & 52 semanas & 48semanas & $\begin{array}{l}\text { IFN 2b 9UM + } \\
\text { LAM (100mg); } \\
\text { LAM (100mg) }\end{array}$ & 3 \\
\hline Chan et al. 27 & 2005 & Positivo & 100 & 52 semanas & 8 semanas & $\begin{array}{c}\text { PEG2b + } \\
\text { LAM (100mg); } \\
\text { LAM (100mg) }\end{array}$ & 4 \\
\hline Yao et al. 28 & 2004 & Positivo & 429 & 1 ano & 2 anos & $\begin{array}{l}\text { LAM }(100 \mathrm{mg})[\mathrm{n}=322] \\
\quad \text { placebo }[\mathrm{n}=107]\end{array}$ & 4 \\
\hline
\end{tabular}

PEG2b: interfe-ron peguilado 2b; IFN2b: interferon alfa $2 b$. 
mento e no seguimento 17,18 . Os efeitos adversos mais freqüentes para o interferon convencional em HBeAg negativo foram cefaléia, síndrome $f l u$, mialgia, artralgia, fadiga 17,18. Também foram encontrados sintomas como leucopenia e trombocitopenia ${ }^{19}$, além de anorexia e alopecia 17 .

PEG2a

Os artigos referentes ao PEG2a em pacientes HBeAg positivo demonstraram, ao final de 48 semanas de tratamento, redução dos níveis DNA do HBV com PEG2a + LAM (86\%), LAM (62\%) e PEG2a (52\%). No seguimento de 24 semanas verificou-se redução semelhante nos níveis de DNA do HBV nos grupos: PEG2a + LAM (34\%) e PEG2a (32\%), resultados melhores que no grupo LAM (22\%) 20 . A soroconversão HBeAg foi melhor nos grupos PEG2a (27\%) e PEG2a + LAM (24\%) comparados à LAM (20\%). No seguimento, os valores também foram melhores nos dois primeiros grupos (32\% e 27\%) comparados com $19 \%$ no grupo LAM 20. Comparando PEG2a em três doses $(90 \mu \mathrm{g} / \mathrm{semana}, 180 \mu \mathrm{g} / \mathrm{semana}$ e $270 \mu \mathrm{g} / \mathrm{semana}$ ) com interferon por 24 semanas, obtiveram-se maiores reduções nos níveis de DNA do HBV e soroconversão HBeAg para o grupo PEG2a que interferon, todavia, as diferenças não foram estatisticamente significantes 21 . Quanto aos resultados secundários, constataram-se, ao final do tratamento, maiores reduções em níveis de ALT com LAM (62\%) comparada com PEG2a + LAM (46\%) e PEG2a (39\%), porém, no seguimento PEG2a (41\%) e PEG2a + LAM (39\%), apresentaram melhores resultados comparados à LAM (28\%) 20. Maior redução nos níveis de ALT foi registrada, ao final do segui-

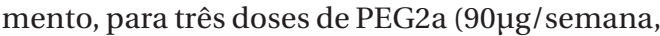
$180 \mu \mathrm{g} /$ semana e $270 \mu \mathrm{g}$ / semana) comparadas ao interferon, sem apresentar, no entanto, diferenças estatisticamente significantes 21 . Melhorias histológicas foram mais vistas nos grupos PEG2a + LAM (41\%) e PEG2a (38\%) do que no grupo LAM (34\%) 20.

Para os pacientes HBeAg negativo, perceberam-se, no tratamento de 48 semanas, que maiores reduções nos níveis DNA do VHB ocorreram com PEG2a + LAM (92\%) e PEG2a (81\%) do que LAM (44\%). No seguimento de 24 semanas, as reduções foram menores, mas melhores resultados também foram obtidos nos grupos de PEG2a + LAM (44\%) e PEG2a (43\%) que no grupo LAM (29\%) 22. Reduções dos níveis de ALT, ao final do tratamento, foram melhores com LAM (73\%) e PEG2a + LAM (49\%) comparadas com PEG2a (38\%). No seguimento, maior redução foi obtida no grupo de PEG2a + LAM (60\%) e PEG2a (59\%), comparada à LAM (44\%) 22. Houve melhorias histológicas semelhantes entre os PEG2a (48\%), PEG2a + LAM (38\%) e LAM (40\%) 22.

A soroconversão HBsAg para os pacientes HBeAg positivo foi de $3 \%$ nos grupos PEG2a e PEG2a + LAM 20. Já nos pacientes HBeAg negativo ela foi de $3 \%$ no grupo PEG2a e $2 \%$ no grupo PEG2a + LAM 22. Não ocorreu soroconversão HBsAg no grupo LAM para as duas categorias de pacientes.

Os efeitos adversos foram mais significativos em PEG2a comparados à LAM. Para os pacientes HBeAg negativo, os efeitos adversos mais citados foram mialgia (26\%-28\% vs. 3\%-6\%), alopecia $(11 \%-29 \%$ vs. $2 \%)$ e anorexia $(13 \%-18 \%$ vs. $2 \%$ ) 20,22. Para os pacientes HBeAg positivo foram mialgia ( $46 \% v s .42 \%$ ) e alopecia ( $44 \% v s$. 24\%) 21 .

\section{LAM}

Para a terapia com a LAM, foram também incluídos estudos em pacientes com complicações como exacerbações agudas graves, cirrose descompensada e transplantes de fígado. Considerou-se o tratamento por pelo menos três meses e o seguimento de pelo menos seis meses. A dosagem usual de LAM foi de $100 \mathrm{mg} /$ dia. Os resultados primários e secundários foram os mesmos enfocados na terapia de interferon e PEG2a, acrescidos do desenvolvimento de variantes resistentes à LAM (mutantes).

Em pacientes HBeAg positivo, em um ano de tratamento, ocorreu soroconversão HBeAg (8\%-44\% vs. $11 \%-12 \%$ ) com manutenção, na maioria dos estudos, dessa soroconversão após dois anos de tratamento em pacientes que não desenvolveram resistência ao medicamento 23,24,25,26,27,28,29. A soroconversão HBeAg foi maior naqueles pacientes com níveis elevados de ALT no pré-tratamento (ALT > 5 vezes o limite normal superior ( $42 \%$ vs. 12\%) 23. Taxas de soroconversão HBeAg foram ainda maiores (50\%) após cinco anos de tratamento contínuo ${ }^{30}$. Modelos multivariados demonstraram que os níveis de ALT no pré-tratamento $(\mathrm{p}<0,001)$ e o índice de atividade histológica ( $p<0,001$ ) foram considerados um importante marcador de prognóstico de queda do HBeAg em asiáticos e caucasianos 23. Soroconversão de DNA do HBV variaram de $10 \%-37 \%$ vs. $16 \%$ e maiores reduções também foram referenciadas com níveis mais elevados de ALT no pré-tratamento 27,29. Soroconversão HBsAg ocorreu em $20 \%$ dos pacientes, após um ano de tratamento 26. Quando os resultados secundários foram analisados, melhorias histológicas foram encontradas após um ano de tratamento, pelo índice de atividade histológica $(15,5 \times 5,5)$, com aproximadamente $70 \%$ de de- 
créscimo de dois pontos nos escores de Knodell ( $\mathrm{p}<0,001$ ) comparado a $20 \%-24 \%$ nos controles 24,25,31. Melhorias histológicas em torno de $59 \%$ também foram referidas, após um ano de tratamento considerando-se acréscimo igual ou superior a dois pontos nos escores de Knodell em necroinflamação 27 . Resistência com mutação YMDD foi percebida em $12 \%-57 \%$ dos pacientes tratados por até dois anos 24,27,28,29,31,32. Identificou-se no seguimento desenvolvimento progressivo da resistência em período acima de quatro anos (71\%-76\%) 24,26.

Nos pacientes HBeAg negativo, negativação de DNA do HBV ocorreu entre 72\%-74\% em 48 semanas de tratamento 33,34. Resposta sustentada, após um ano de tratamento foi observada em $11 \%-20 \%$ dos pacientes 32 . Quando os resultados secundários foram analisados, a normalização dos níveis de ALT foram em torno de 70\%-80\% em 48 semanas de tratamento 33,35. Encontrou-se associação entre presença de mutação e piores escores histológicos ( $\mathrm{p}=0,001)$ em 19\% dos pacientes, e também a níveis mais elevados de ALT e DNA do HBV durante o tratamento ${ }^{35}$. Resistência de $16 \%-25 \%$ foi registrada após dois anos de tratamento 33,35 .

A resistência genotípica foi descrita em 25\% dos pacientes após dois anos de tratamento e $44 \%$ apresentaram reagudização (flares) 33 .

Estudo em transplantados de fígado comparando imunoglobulina (HBIg) e LAM descreveu associação da mutação com o aumento dos níveis de DNA do HBV e ALT, que foram ligeiramente mais elevados no grupo de LAM. Não houve recorrência do vírus da hepatite $\mathrm{B}$ em ambos os grupos 36 . Redução dos níveis DNA do HBV e normalização da ALT foram relatadas em três meses de tratamento e $50 \%$ desenvolveram carcinoma hepatocelular $(\mathrm{p}=0,013) 37$. Resposta virológica mostrou a mutação YMDD de 54\%-59\% 37,38.

Os efeitos adversos mais citados foram dor de cabeça ( $25 \%$ vs. $17 \%)$, fadiga (17\%) e náusea (17\% vs. 8\%) 36; fadiga (20\%) e depressão (13\%) 26; e artralgia (13\%) 38. A LAM apresentou boa eficácia e foi bem tolerada nos três primeiros anos de tratamento 31 .

\section{Discussão}

O benefício da utilização do interferon convencional foi alcançado em 1/3 dos pacientes tratados, em 4 a 6 meses de tratamento, para os pacientes HBeAg positivos, não tendo sido registradas vantagens no prolongamento do tempo de tratamento. Como desvantagens observaram-se limitações de utilização em pacientes com baixos níveis de ALT no pré-tratamento e nos HBeAg ne- gativos, devido à ocorrência de recaídas e efeitos colaterais, além da inconveniência da administração subcutânea do medicamento. Doses mais elevadas do interferon apresentaram melhores resultados, mas foram associadas ao aumento da freqüência e à gravidade dos efeitos colaterais. Considerando a qualidade metodológica dos artigos revisados, classificada como " baixa a moderada”, deve-se ter cautela quanto à evidência dos resultados observados, uma vez que essa qualidade é relevante para a revisão sistemática e pode influenciar a magnitude desses resultados.

Resultados semelhantes foram relatados em metanálise de 15 estudos randomizados e controlados com o interferon convencional em pacientes HBeAg positivo. Constatou-se que a perda de HBeAg e DNA do HBV também foi limitada, sendo observada em $33 \%$ e $37 \%$ dos tratados e $12 \%$ e $17 \%$ dos controles, respectivamente. Altos níveis de ALT no pré-tratamento também foram preditivos de boa resposta a essa medicação 39 .

Na maioria dos estudos, em pacientes HBeAg positivo, resposta virológica sustentada foi observada em cerca de 1/3 dos pacientes tratados com PEG2a. Em pacientes HBeAg positivo tratados com PEG2a, no seguimento, a redução dos níveis de DNA do HBV e de ALT foi maior e a soroconversão HBeAg foi melhor quando comparados com pacientes tratados com LAM. O PEG2a também mostrou melhores taxas de resposta que interferon, no seguimento, mas as diferenças não foram estatisticamente significantes. Para os pacientes HBeAg negativo, no tratamento e no seguimento, maiores reduções nos níveis DNA do HBV ocorreram nos grupos PEG2a comparados ao LAM. A redução dos níveis de ALT, no seguimento, também foram maiores nos grupos PEG2a. As taxas de resposta observadas foram mais elevadas para os pacientes HBeAg negativo.

Soroconversão HBsAg não foi reportada para o grupo de LAM. Os estudos com PEG2a relataram efeitos colaterais semelhantes ao interferon convencional, com o benefício adicional de administração uma vez por semana. Comparados à LAM, os efeitos colaterais foram mais significativos. Como o PEG2a é uma intervenção relativamente nova para o tratamento da hepatite crônica $\mathrm{B}$, ainda não há evidências sobre a eficácia do medicamento para o tratamento de pacientes com cirrose e diferentes grupos étnicos, nem quanto aos efeitos de tratamento a longo prazo e durabilidade de resposta após término do tratamento. Questões como superioridade de eficácia do PEG2a comparada ao interferon convencional não apresentaram respostas conclusivas, uma vez as diferenças encontradas não foram estatisticamente significantes. Preditores de resposta para o interferon convencional e 
LAM incluem elevados níveis de ALT no pré tratamento e baixos níveis de DNA do HBV, contudo mais pesquisas são necessárias para avaliar se os mesmos se aplicam ao PEG2a. A qualidade metodológica de todos os artigos revisados enfocando PEG2a foi classificada como "moderada". Para assegurar maior evidência são necessários estudos de melhor qualidade metodológica, que comparem os resultados do PEG2a com o interferon convencional, que avaliem a duração ideal de tratamento, além da definição dos preditores de resposta e resultados de tratamento em diferentes grupos étnicos.

Em metanálise de estudos randomizados e controlados em pacientes HBeAg positivo utilizando o PEG2a, e com predominância de pacientes asiáticos, os grupos PEG2a tiveram resultados superiores aos LAM, induzindo resposta bioquímica sustentada e resposta virológica em um terço dos pacientes após 48 semanas de terapia. $\mathrm{O}$ PEG2a foi bem tolerado e efeitos colaterais mais sérios foram reportados em menos de $10 \%$ dos pacientes 40.

A lamivudina apresentou resposta virológica inicial ou supressão bioquímica. O mais importante preditor de resposta favorável ao tratamento com LAM foi relacionado aos níveis elevados de ALT no pré-tratamento com maiores taxas de soroconversão HBeAg, variando de $8 \%$ para pacientes com níveis de ALT $<2$ vezes o limite superior da normalidade para $42 \%$ com níveis de ALT $>5$ vezes o limite superior da normalidade. A manutenção de resposta contudo foi fraca, principalmente em pacientes HBeAg negativo, os quais requerem tratamento por tempo mais prolongado, aumentando o risco de seleção de mutantes resistentes à lamivudina, diminuindo as taxas de resposta ao longo do tempo e elevando o risco de progressão da doença. O desenvolvimento de resistência demonstrou-se progressivo com o tempo de tratamento, aumentando de $12 \%-57 \%$ em dois anos para $71 \%-76 \%$ acima de quatro anos. Melhorias histológicas foram observadas no tratamento com LAM, contudo presença de mutação foi associada com piores escores histológicos. De maneira geral, a LAM revelou efeitos colaterais mínimos, evidenciando boa eficácia e tolerância. A qualidade metodológica dos artigos revisados enfocando LAM apresentou classificação "moderada a alta" demonstrando melhor nível de evidência científica que os estudos sobre interferons.

Revisão sistemática enfocando a LAM em pacientes HBeAg positivo e negativo também demonstrou boa resposta virológica inicial ou supressão bioquímica. Taxas mais altas de resposta virológica duradoura foram encontradas em pacientes sem resistência viral, sendo que es- ses resultados foram melhores para os pacientes HBeAg positivo (24\%) 41.

Uma das limitações desse estudo diz respeito à carência de estudos nacionais que abordem o tratamento da hepatite crônica B. Devido a isso, as principais fontes de informação foram estudos internacionais, realizados predominantemente na Ásia e Extremo Oriente com genótipos B e C. Análises futuras são necessárias para verificar se estes achados se repetirão em populações de diferentes grupos étnicos e genótipos. Também é importante mencionar a discussão recente na literatura quanto à relevância dos diferentes genótipos do HBV na eficácia do tratamento e no prognóstico da doença 42. Entretanto, as evidências disponíveis até o momento não permitem inserir a genotipagem como um dos critérios de decisão rotineiros para a abordagem dos pacientes infectados pelo HBV 43. É necessário um acúmulo maior de conhecimento para definir a genotipagem do HBV como um fator relevante na decisão da terapêutica e no seguimento clínico de pacientes com hepatite $B$.

\section{Conclusão}

O conhecimento sobre a hepatite crônica B e suas opções terapêuticas tem se incrementado ao longo do tempo. Entretanto, muitos aspectos sobre a doença ainda permanecem desconhecidos e as alternativas medicamentosas estão longe de serem consideradas ideais. As vantagens demonstradas pelo interferon foram resposta duradoura em HBeAg positivos, tempo de duração de tratamento curto (6 a 12 meses) e o não surgimento de resistência. As desvantagens foram os efeitos colaterais e a via de administração, que podem afetar negativamente a adesão ao tratamento, além da limitação de sucesso para pacientes HBeAg negativo. O PEG2a apresenta como vantagens a administração semanal e a ausência de resistência à terapêutica e como desvantagens a via de administração e os efeitos adversos, além da contra-indicação em pacientes com doença hepática descompensada. Questões como a definição do tempo ideal de tratamento e a determinação mais apurada de preditores de resposta ainda não estão resolvidas. Para a LAM foram demonstradas vantagens como a facilidade de administração (via oral), rápida inibição da replicação viral, ótima tolerância e capacidade de utilização em pacientes com cirrose hepática descompensada ou em pré e pós-transplante hepático. As desvantagens identificadas com relação à LAM foram as recaídas e altas taxas de resistência viral do tipo YMDD, além da necessidade de terapia prolongada (mínimo 12 meses). 
O desenvolvimento de antivirais mais potentes, a utilização de novas associações de medicamentos e a melhor compreensão dos mecanismos de resistência do HBV são importantes fatores a se considerar na busca de melhor eficácia do tratamento e da diminuição, no futuro, da carga global de portadores do HBV. Um número expressivo de análogos de nucleosídeos/ nucleotídeos estão atualmente disponíveis para o tratamento da hepatite B. Sua utilização, ain- da recente, mostra tendência ao crescimento e requer estudos que avaliem a eficácia e a efetividade desses novos medicamentos no controle da replicação do HBV. O acúmulo e a adequada sistematização deste conhecimento ampliarão a qualidade da evidência existente, configurando um subsídio fundamental ao processo de decisão sobre a manutenção e/ou incorporação de medicamentos nos protocolos clínicos para o tratamento da hepatite viral crônica B.

\section{Resumo}

A hepatite crônica B constitui um grave problema de saúde pública e vem demonstrando crescentes gastos com financiamento de medicamentos de dispensação em caráter excepcional e de alto custo no Sistema Único de Saúde (SUS). O objetivo do estudo foi comparar a eficácia do interferon (convencional; peguilado - PEG2a) e lamivudina (LAM) para o tratamento da hepatite crônica B, pelo método de revisão sistemática selecionando ensaios clínicos randomizados e controlados identificados nas bases PubMed e LILACS. As medidas de resultado consideradas foram resposta virológica, soroconversão, resposta bioquímica, resposta histológica e efeitos adversos. Foram selecionados 35 artigos. A presença ou ausência do HBeAg e os níveis de alanina amino transferase (ALT) no pré-tratamento demonstraram papel fundamental na indicação terapêutica inicial. O tratamento com interferons convencionais permite a inativação da doença por longos períodos de tempo, podendo resultar em soroconversão HBsAg. O PEG 2 a demonstrou eficácia superior ao interferon e LAM e efeitos colaterais semelhantes ao interferon. A LAM apresenta vantagem de ser sensivel para os pacientes HBeAg negativo e apresenta como maior desvantagem o desenvolvimento de resistência.

\section{Colaboradores}

A. M. Almeida participou do planejamento e elaboração do estudo, seleção dos artigos, revisão bibliográfica, avaliação metodológica e redação do artigo. D. I. Silva participou da seleção dos artigos, avaliação metodológica e redação final do artigo. A. A. Guerra Jr. e G. D. Silva participaram da elaboração do protocolo de revisão sistemática e redação final do artigo. F. A. Acurcio participou do planejamento, elaboração do estudo e redação do artigo.

\section{Agradecimentos}

Os autores agradecem aos integrantes do Grupo de Pesquisa em Farmacoepidemiologia da Universidade Federal de Minas Gerais por sua valorosa contribuição desde o desenho metodológico à revisão final. Este estudo é parte integrante do projeto de pesquisa Avaliação Farmacoeconômica e Epidemiológica do Programa de Medicamentos Excepcionais do SUS que obteve financiamento do Conselho Nacional de Desenvolvimento Científico e Tecnológico (CNPq, processo 402691/ 2005-9) e da Fundação de Amparo à Pesquisa do Estado de Minas Gerais (FAPEMIG, processo 4611-5.01/07).

Hepatite B Crônica; Interferons; Lamivudina; Resultado de Tratamento 


\section{Referências}

1. Leemans WF, Janssen HLA, De Man RA. Future prospective for the management of chronic hepatitis. World J Gastroenterol 2007; 13:2554-67.

2. Wright TL. Introduction to chronic hepatitis b infection. Am J Gastroenterol 2006; 101 Suppl 1: S1-6.

3. Lee WM. Hepatitis B virus infection. New Engl J Med 1997; 337:1733-45.

4. Shepherd J, Jones J, Takeda A, Davidson P, Price A. Adefovir dipivoxil and pegylated interferon alfa-2a for the treatment of chronic hepatitis B: a systematic review and economic evaluation. Health Technol Assess 2006; 10: iii-iv, xi-xiv, 1-183.

5. Secretaria de Atenção à Saúde, Ministério da Saúde. Portaria n ${ }^{\circ}$. 860, de 04 de novembro de 2002. Aprova o Protocolo Clínico e Diretrizes Terapêuticas - Hepatite Viral Crônica B. Diário Oficial da União 2002, 5 nov.

6. Woodroffe R, Yao GL, Meads C, Bayliss S, Ready A, Raftery J, et al. Clinical and cost-effectiveness of newer immunosuppressive regimens in renal transplantation: a systematic review and modelling study. Health Technol Assess 2005; 9:1-179, iii-iv.

7. Carreño V, Porres JC, Mora I, Gutiez J, Quiroga JA, Ramon Y, et al. A controlled study of treatment with recombinant interferon alpha in chronic hepatitis B virus infection: indution and maintenance schedules. Antiviral Res 1987; 8:125-37.

8. Di Bisceglie AM, Fong TL, Fried MW, Swain MG, Baker B, Korenman J, et al. A randomised controlled trial of recombinant alfa-interferon therapy for chronic hepatitis B. Am J Gastroenterol 1993; 88:1887-92

9. Lok ASF, Lai CL, Wu PC, Leung EKY. Long-term follow-up in a randomized controlled trial of recombinant alpha 2-interferon in Chinese patients with chronic hepatitis B infection. Lancet 1988; 2:298302.

10. Müller R, Baumgarten R, Markus R, Schulz M, Wittenberg H, Hintsche-Kilger B, et al. Treatment of chronic hepatitis B with interferon alfa-2b. J Hepatol 1990; 11 Suppl 1:S137-40.

11. Sarin SK, Guptan RC, Thakur V, Malhotra S, Malhotra V, Banerjee K, et al. Efficacy of low-dose alpha interferon therapy in HBV-related chronic liver disease in Asian Indians: a randomised controlled trial. J Hepatol 1996; 24:391-6.

12. Pastore G, Santantonio T, Milella M, Monno L, Mariano N, Moschetta R, et al. Anti-HBe-positive chronic hepatitis B with HBV-DNA in the serum response to a 6-month course of lymphoblastoid interferon. J Hepatol 1992; 14:221-5.

13. Porres JC, Carreño V, Mora I, Gutiez J, Moreno A, Ramon Y, et al. Different doses of recombinant alpha interferon in the treatment of chronic hepatitis B patients without antibodies against the human immunodeficiency virus. Hepatogastroenterology 1988; 35:300-3.

14. Carreño V, Marcelin P, Hadziyannis S, Salmerón J, Diago M, Kitis GE, et al. Retreatment of chronic hepatitis B e antigen-positive patients with recombinant interferon alfa-2a: the European Concerted Action on Viral Hepatitis(EUROHEP). Hepatology 1999; 30:277-82.
15. Perrillo RP, Schiff ER, Davis GL, Bodenheimer Jr. HC, Lindsay K, Payne J, et al. A randomised, controlled trial of interferon alfa-2b alone and after prednisone withdrawal for the treatment of chronic hepatitis B: the Hepatitis Interventional Therapy Group. N Engl J Med 1990; 323:295-301.

16. Thomas HC, Lok ASF, Carreño V, Farrel G, Tanno $\mathrm{H}$, Perez V, et al. Comparative study of three doses of interferon-alpha 2a in chronic active hepatitis B: the International Hepatitis Trial Group. J Viral Hepat 1994; 1:139-48.

17. Fattovich G, Farci P, Rugge M, Brollo L, Mandas A, Pontisso P, et al. A randomised controlled trials of lymphoblastoid interferon-alfa in patients with chronic hepatitis B lacking HBeAg. Hepatology 1992; 15:584-9

18. Lampertico P, Del NE, Manzin A, Donato MF, Rumi MG, Lunghi G, et al. A randomized, controlled trial of a 24-month course of interferon alfa $2 \mathrm{~b}$ in patients with chronic hepatitis B who had hepatitis B virus DNA without hepatitis B e antigen in serum. Hepatology 1997; 26:1621-5.

19. Hadziyannis S, Bramou T, Makris A, Moussoulis G, Zignego L, Papaioannou C. Interferon alfa-2b treatment of HBeAg negative/serun HBV DNA positive chronic active hepaitis type B. J Hepatol 1990; 11 Suppl 1:S133-6.

20. Lau GKK, Piratvisuth T, Luo KX, Marcellin P, Thongasawat S, Cooksley G, et al. Peginterferon alfa2a, lamivudine, and the combination for HBeAgpositive chronic hepatitis B. N Engl J Med 2005; 352:2682-95.

21. Cooksley WG, Piratvisuth T, Lee SD, Mahachai V, Chao YC, Tanwandee T, et al. Peginterferon alpha$2 \mathrm{a}(40 \mathrm{kDa})$ : an advance in the treatment of hepatitis B e antigen-positive chronic hepatitis B. JViral Hepat 2003; 10:298-305.

22. Marcellin P, Lau GK, Bonino F, Farci P, Hadziyannis S, Jin R, et al. Peginterferon alfa-2a alone, lamivudine alone, and the two in combination. N Engl J Med 2004; 351:1206-17.

23. Perrillo RP, Lai CL, Liaw YF, Dienstag J, Schiff ER, Schalm SW, et al. Predictos of HbeAg loss after lamivudine treatment for chronic hepatitis B. Hepatology 2002; 36:186-94.

24. Lau DT, Khokhar MF, Doo E, Ghany MG, Park Y, Kleiner DE, et al. Long-term therapy of chronic hepatitis B with lamivudine. Hepatology 2000; 32(4 Pt 1):828-34.

25. Buti M, Cotrina M, Jardi R, Castro EC, RodriguezFrias F, Sànchez-Avila F, et al. Two years of lamivudine therapy in anti-Hbe-positive patients with chronic hepatitis B. JViral Hepat 2001; 8:270-5.

26. Dienstag JL, Cianciara J, Karavalcin S, Kowdley KV, Willems B, Plisek S, et al. Durability of serologic response after lamivudine treatment of chronic hepatitis B. Hepatology 2003; 37:748-55.

27. Chan HL, Leung NW, Hui AY, Wong VW, Liew C, Chim AM, et al. A randomized, controlled trial of combination therapy for chronic hepatitis B: comparing pegylated interferon-alpha $2 \mathrm{~b}$ and lamivudine with lamivudine alone. Ann Intern Med 2005; 142:240-50. 
28. Yao GB, Cui ZY, Wang BE, Yao JL, Zeng MD. A 3-year clinical trial of lamivudine in treatment of patients with chronic hepatitis B. Hepatobiliary Pancreat Dis Int 2004; 3:188-93.

29. Barbaro G, Zechini F, Pellicelli AM, Francavilla R, Scotto G, Bacca D, et al. Long-term efficacy of interferon alpha- $2 \mathrm{~b}$ and lamivudine in combination compared to lamivudine monotherapy in patients with chronic hepatitis B. An Italian multicenter, randomized trial. J Hepatol 2001; 35:406-11.

30. Lok ASF, Ching-Lung L, Leung N, Guang-Bi Y, Zhen-Yu C, Eugene R, et al. Long-term safety of lamivudine treatment in patients with chronic hepatitis B. Gastroenterology 2003; 125:1714-22.

31. Leung NWY, Ching-Lung LAI, Ting-Tsung C, Richard G, Chuan-Mo L, Keng-Yeen NG, et al. Extended lamivudine treatment in patients with chronic hepatitis B enhances hepatitis B e antigen seroconversion rates: results after 3 years of therapy. Hepatology 2001; 33:1527-32.

32. Manolakopoulos S, Karatapanis S, Elefsiniotis J, Mathou N, Vlachogiannakos J, Iliadou E, et al. Clinical course of lamivudine monotherapy in patients with descompensated cirrhosis due to HBeAg negative chronic HBV infection. Am J Gastroenterol 2004; 99:57-63.

33. Fung SKF, Wong M, Hussain D, Lok ASF. Sutained response after a 2-year course of lamivudine treatment of hepatitis B e antigen-negative chronic hepatitis B. J Viral Hepat 2004; 11:432-8.

34. Lai CL, Chien RN, Leung NW, Chang TT, Guan $\mathrm{R}$, Tai DI, et al. A one-year trialof lamivudine for chronic hepatitis B. Asia Hepatitis Lamivudine Study Group. N Engl J Med 1998; 339:61-8.

35. Rizzetto M, Tassopoulos NC, Goldin RD, Santantonio R, Heathcote EJ, Lagget $\mathrm{M}$, et al. Extended lamivudine treatment in patients with HbeAg-negative chronic hepatitis B. J Hepatol 2005; 42:173-9.

36. Naoumov NV, Lopes AR, Burra P, Caccamo L, Iemmolorm DE, Man RA, et al. Randomized trial of lamivudine versus hepatitis B immunoglobulin for long-term prophylaxis of hepatic B recurrence after liver transplantation. J Hepatol 2001; 34:888-94.

37. Andreone P, Gramenzi AC, Cursaro M, Biselli C, Trevisan CF, Bernardi M. High risk of hepatocellular carcinoma in anti-HBe positive liver cirrhosis patients developing lamivudine resistence. J Viral Hepat 2004; 11:439-42.
38. Fontana RJ, Han HW, Wright T, Everson G, Baker A, Schiff ER, et al. A multicenter study of lamivudine treatment in 33 patients WITH hepatitis B after liver transplantation. Liver Transpl 2001; 7:504-10.

39. Wong DK, Cheung AM, O'Rourke K, Naylor CD, Detsky AS, Heathcote J, et al. Effect of alpha-interferon treatment in patients with hepatitis B e antigen-positive chronic hepatitis B. A meta-analysis. Ann Intern Med 1993; 119:312-23.

40. Hui AY, Chan HL, Cheung AY, Cooksley G, Sung JJ. Systematic review: treatment of chronic hepatitis $B$ virus infection by pegylated interferon. Aliment Pharmacol Ther 2005; 22:519-28.

41. Kanwal F, Gralnek IM, Martin P, Dulai GS, Farid M, Brennan SMR. Treatment alternatives for chronic hepatitis $b$ virus infection: a cost-effectiveness analysis. Ann Intern Med 2005; 142:821-31.

42. Fonseca JCF. História natural da hepatite crônica B. Rev Soc Bras Med Trop 2007; 40:672-7.

43. Sociedade Brasileira de Infectologia. 1o Consenso da Sociedade Brasileira de Infectologia para o diagnóstico e manuseio da hepatite B (e delta). Braz J Infect Dis 2006; 10 Suppl 1:1-78.

44. Krogsgaard K. The long-term effect of treatment with interferon-alpha $2 \mathrm{a}$ in chronic hepatitis B. The Long-Term Follow-up Investigator Group. The European Study Group on Viral Hepatitis (EUROHEP). Executive Team on Anti-Viral Treatment. J Viral Hepat 1998; 5:389-97.

45. Fattovich G, Brollo L, Boscaro S, Pontisso P, Giustina G, Criscuolo D, et al. Long-term effect of low dose recombinant interferon therapy in patients with chronic hepatitis B. J Hepatol 1989; 9:331-7.

46. Realdi G, Fattovich G, Pastore G, Caredda F, Noventa F, Santantonio T, et al. Problems in the management of chronic hepatitis $B$ with interferon: experience in a randomized, multicentre study. J Hepatol 1990; 11 Suppl 1:S129-32.

47. Harry LAJ, Guido G, Carreño V, Marcellin P, Nikolai $\mathrm{V}$, Naoumov AC, et al. Interferon alfa for chronic hepatitis B infection: increased efficacy of prolonged treatment. The European Concerted Action on Viral Hepatitis (EUROHEP). Hepatology 1999; 30:238-43.

48. Lau DTY, Everhart J, Kleiner DK, Park Y, Vergalla J, Schmid P, et al. Long-term follow-up of patients with chronic hepatitis B treated with interferon alfa. Gastroenterology 1997; 113:1660-7.

Recebido em 25/Abr/2008

Versão final reapresentada em 11/Dez/2008

Aprovado em 16/Fev/2009 